\title{
Communications
}

\section{Examen de spécialiste}

Examen de spécialiste pour l'obtention de la formation approfondie en psychiatrie et psychothérapie de la personne âgée en complément de titre de spécialiste en psychiatrie et psychothérapie deuxième partie (oral)

Lieu: Hôpital de psychiatrie de l'âge avancé, Cite de Cery, 1008 Prilly

Date: vendredi, 8 mai 2015

Délai d'inscription: le 31 mars 2015

Vous trouverez de plus amples informations sur le site web de l'ISFM www.siwf.ch $\rightarrow$ Domaines spécialisés $\rightarrow$ Titres de spécialiste et formations approfondies (formation postgraduée) $\rightarrow$ psychiatrie et psychothérapie

\section{Swiss Medical Board}

\section{Fusion du Swiss Medical Board et de SwissHTA}

$\mathrm{Au}$ début de cette année, les organes responsables du Swiss Medical Board (SMB) et de SwissHTA ont engagé un processus de convergence afin de réunir les deux systèmes d'évaluation de l'utilité des prestations médicales. La fusion, basée sur des jalons et des structures définis de concert, sera effective à partir de janvier 2015, sous la houlette du Swiss Medical Board. Ainsi, la Suisse disposera d'une institution HTA solidement ancrée, dont la mission est d'évaluer les prestations médicales sur des bases scientifiques.

Divers acteurs du système de santé se sont engagés, ces dernières années, pour le développement de Health Technology Assessments (HTA) en Suisse. Ce faisant, il s'agit d'évaluer l'utilité de prestations médicales, qu'elles soient récentes ou établies de longue date. De ces initiatives sont nés, d'une part le SMB, une organisation pionnière fondée par le département de la santé du canton de Zurich qui a publié douze rapports HTA depuis 2008 et, d'autre part, le projet SwissHTA dont l'objectif est de promouvoir les processus HTA et de développer des méthodes dans ce sens. Dans le cadre de ce processus de convergence, les meilleurs éléments des deux structures sont réunis pour élaborer une base commune à l'évaluation de l'utilité des prestations médicales, en partant des expériences et de la pratique du Swiss Medical Board et des documents de stratégie et de mise en œuvre de SwissHTA.

L'association des organes responsables de SwissHTA et de l'organe responsable du SMB, à partir de janvier 2015, constitue une partie de ce processus de convergence. Ainsi, la Conférence des directeurs de la santé (CDS), l'Académie Suisse des Sciences Médicales (ASSM), la Fédération des médecins suisses (FMH), le gouvernement de la Principauté du Liechtenstein et dorénavant l'association des assurancesmaladies curafutura et santésuisse de même qu'Interpharma sont coresponsables du développement institutionnel, structurel et méthodologique; ils sont également chargés de faire élaborer et publier les rapports HTA.

Grâce au regroupement du know-how et à la base solide, le SMB élargi a de grandes chances d'établir à long terme, en Suisse, une institution HTA acceptée tant au niveau professionnel que politique.

\section{Ligue suisse contre le cancer}

\section{Rapport de recherche 2013: 20 millions de francs pour la recherche universitaire}

L'an passé, la fondation Recherche suisse contre le cancer, la Ligue suisse contre le cancer et les ligues cantonales et régionales ont soutenu la recherche universitaire sur le cancer avec un nouveau montant record de plus de 20 millions de francs. Plus de $80 \%$ de cette somme sont allés à des projets de recherche indépendants. Le nouveau rapport de recherche 2013 docu- mente clairement la manière dont les dons sont utilisés pour soutenir la recherche sur le cancer. On y présente les résultats des projets qui ont été achevés en 2013, ainsi que les objectifs de ceux approuvés au cours de cette période. Le rapport peut être commandé gratuitement en français, en allemand et en anglais par courriel à l'adresse barbara.messerli[at]liguecancer.ch ou par téléphone au numéro 0337736557.

\section{FASMED}

FASMED unterstützt Kompetenzzentrum für translationale Medizin und Unternehmertum

Der FASMED befürwortet, dass mit einem nationalen Technologie-Kompetenzzentrum in Bern die Zusammenarbeit zwischen Industrie und forschenden Klinikern erleichtert und die Rahmenbedingungen für die Translation im globalen Standortwettbewerb verbessert werden sollen.

Der Aufbau eines nationalen Kompetenzzentrums für Translationale Medizin und Unternehmertum wurde vom Regierungsrat initiiert. Es soll dazu beitragen, den Übergang der Erkenntnisse der medizinischen Forschung und industriellen Entwicklung in die klinische Anwendung («Translation») zu fördern. Mit dieser neuen, prozessorientierten Disziplin soll die Interaktion zwischen präklinisch tätigen Forschern, Klinikern, regulatorischen Instanzen und Investoren professionalisiert werden. Die Translationale Medizin soll dazu die Entwicklung neuer Technologien und Produkte beschleunigen helfen.

Der FASMED setzt sich für die Wahrung des raschen und unbürokratischen Zugangs der Patienten zu Innovationen ein und unterstützt daher die Ziele des nationalen Kompetenzzentrums vollumfänglich. Dazu wird er die weitere Entwicklung verfolgen und sich womöglich unterstützend und beratend mit einbringen. 